\title{
L'AMÉNAGEMENT DU DANUBE ALLEMAND DANS L'INTÉRÊT DU DÉVELOPPEMENT DU PAYS ET DE LA NAVIGATION FLUVIALE
}

Si l'on admet que l'origine du Danube se situe à la puissante source qui jaillit près de Donaueschingen, et à quelque distance à l'amont du confluent des deux torrents Brigach et Brege, qui donnent naissance au fleuve proprement dit, on peut considérer que le cours allemand du Danube entre Donaueschingen et Jochenstein (à la frontière autrichienne) se compose de trois tronçons distincts, suivant ses débits liquides et solides, les aménagements réalisés, et ses conditions de navigation et administratives. De ces trois parcours:

-...- le premier s'étend de Donaueschingen jusqu'au confluent de l'Iller à $7,7 \mathrm{~km}$ en amont de la ville d'Ulm;

- le deuxième s'étend de l'Iller jusqu'au débouché du canal «Ludwig-Donau-Main » à Kelheim;

- le troisième enfin s'étend de Kelheim à la frontière autrichienne à Jochenstein.

\section{1.}

\section{Le Danube entre Donaueschingen et I'lller}

Ce parcours s'étend sur $194 \mathrm{~km}$, et se situe à présent entièrement dans le pays de Bade-Wurlemberg.

Les eaux du Danube proviennent, par les deux torrents Brigach et Brege, de la roche primitive de la Forêt Noire, traversent ensuite des formations de calcaire conchylien et de marne irisée, percent le Jura Blanc à deux endroits, chaque fois par une vallée étroite et encaissée entre les monlagnes, et

* Directeur des Eaux et de la Navigation a Ratisbonne. enfin, s'écoulent sur des terrains constitués de molasses plus tendres, et de moraines de glaciers alpins. Dans les vallées plus larges à l'aval, le fleuve s'écoule sur un fond de sable et de gravier en formant de nombreuses boucles et méandres, dont les berges étaient attaquées sans cesse par l'érosion, avant qu'il n'y fût apporté les ouvrages de protection indispensables. Les terres agricoles riveraines se trouvaient menacées de plus en plus par les dépôts constitués de matériaux arrachés de ces berges, et c'était justement à l'époque de la moisson que se faisaient sentir chaque année les effets néfastes des crues d'été. Dans ces conditions, les communes riveraines ont été obligées, presque dès le début du $\mathrm{x}_{\mathrm{IX}}{ }^{\circ}$ siècle, à prendre des mesures de leur propre chef, et de solliciter l'aide de l'Etat pour la réalisation d'ouvrages de protection des berges et de correction du cours du fleuve. C'est ainsi qu'il a été construit, dans la partie aval du cours entre Scheer et Ulm (situé autrefois sur le territoire du Wurtemberg), à partir de l'année 1820 et jusqu'à la fin du Xix ${ }^{\mathrm{e}}$ siècle, des ouvrages de protection et de régularisation coûtant environ 1,5 million de Marks (valeur de l'époque). On s'était très tôt fixé, pour le chenal des moyennes eaux, des largeurs de régularisation normalisées allant d'environ 25 à $50 \mathrm{~m}$, juste à l'amont du confluent de l'Iller. Par ailleurs, on fait participer le fleuve lui-même à la réalisation des nombreuses coupures nécessaires pour la correction de ses méandres. Les ouvrages de fermeture et de guidage ont surtout été construits sous la forme de fascinages assurés par des enrochements. Le chenal d'écoulement des eaux moyennes ainsi créé (le débit des eaux moyennes à l'amont du débouché de l'Iller est de l'ordre de $35 \mathrm{~m}^{3} / \mathrm{s}$ ) a permis au fleuve d'évacuer, sur l'ensem- 


\section{F. DOBMAYER.}

ble de cette partie de son cours, même certains débits de crue d'ordre mineur en toute sécurité, grâce au redressement de son tracé, et au raidissement de sa pente d'environ $1,3 \%$ - $0,8 \%$.

L'endiguement des débits de crue a fait l'objet d'études approfondies. On s'est décidé, dans la plupart des cas, à ne pas réaliser un endiguement complet pour contenir les eaux de crue, ce qui aurait nécessité des écartements de profils allant jusqu'à $200 \mathrm{~m}$; on s'est simplement contenté, soit de prévoir localement des digues d'été, soit de surélever les berges autant que nécessaire. Avant l'aménagement du fleuve, la largeur du lit majeur lors des plus grandes crues avait été variable, pouvant atteindre plus de $1000 \mathrm{~m}$ dans certaines parties du cours aval.

Pratiquement, l'ensemble du cours du Danube, jusqu'au confluent de l'Iller, était déjà aménagé de la manière décrite dès le début $d u x^{\circ}$ siècle. La construction de trois centrales hydroélectriques à Wiblingen, Donaustetten et Opfingen (à l'amont de la ville d'Ulm) pendant les années 1930 a profondément modifié les 25 derniers kilomètres environ du cours.

Etant donné qu'au $\mathrm{xIx}^{\mathrm{e}}$ siècle la navigation fluviale ne commencait qu'à partir d'Ulm, et que l'on ne pratiquait mème pas le flottage à l'amont de cette ville, aucune mesure n'était nécessaire sur cette partie du cours. On trouvait à l'époque de nombreux moulins et usines employant les eaux du fleuve sur l'ensemble de ce parcours.
2.

\section{Le Danube}

\section{entre le confluent de l'lller et le débouché} du canal « Ludwig-Donau-Main "

L'ensemble de ce parcours est situé en Bavière, à l'exception toutefois des premiers $7,7 \mathrm{~km}$ de la rive gauche, à partir du débouché de l'Yller, qui se trouvent sur le territoire du pays de Bade-Wurtemberg. Toutes les mesures de régularisation du fleuve, sauf celles lançées en premier lieu par des communes ou des localités riveraines, ont été réalisées (et le sont encore aujourd'hui) par l'Administration Bavaroise des Travaux publics.

Entre Ulm et Kelheim, le cours du fleuve s'oriente vers le nord-est, en s'écoulant entre le Jura souabe et franconien d'un côté, et les coteaux du hautplateau souabe-bavarois de l'autre, et perce les derniers contreforts du Jura à deux endroits, dont l'un se situe entre Stepperg et Neuburg, et l'autre entre Neustadt et Regensburg. Cependant, sur de loin la plus grande partie de ce parcours, le fleuve s'écoule par des larges fonds de vallée entre les berges de faible hauteur.

A partir d'Ulm, le régime du fleuve subit l'influence prépondérante de ses puissants affluents alpins, l'lller et le Lech. Par leurs très importants apports de matériaux solides, ces deux rivières confèrent au Danube toutes les caractéristiques d'un fleuve transportant des matériaux solides. La superficie du bassin versant passe de $8000 \mathrm{~km}^{2}$ à

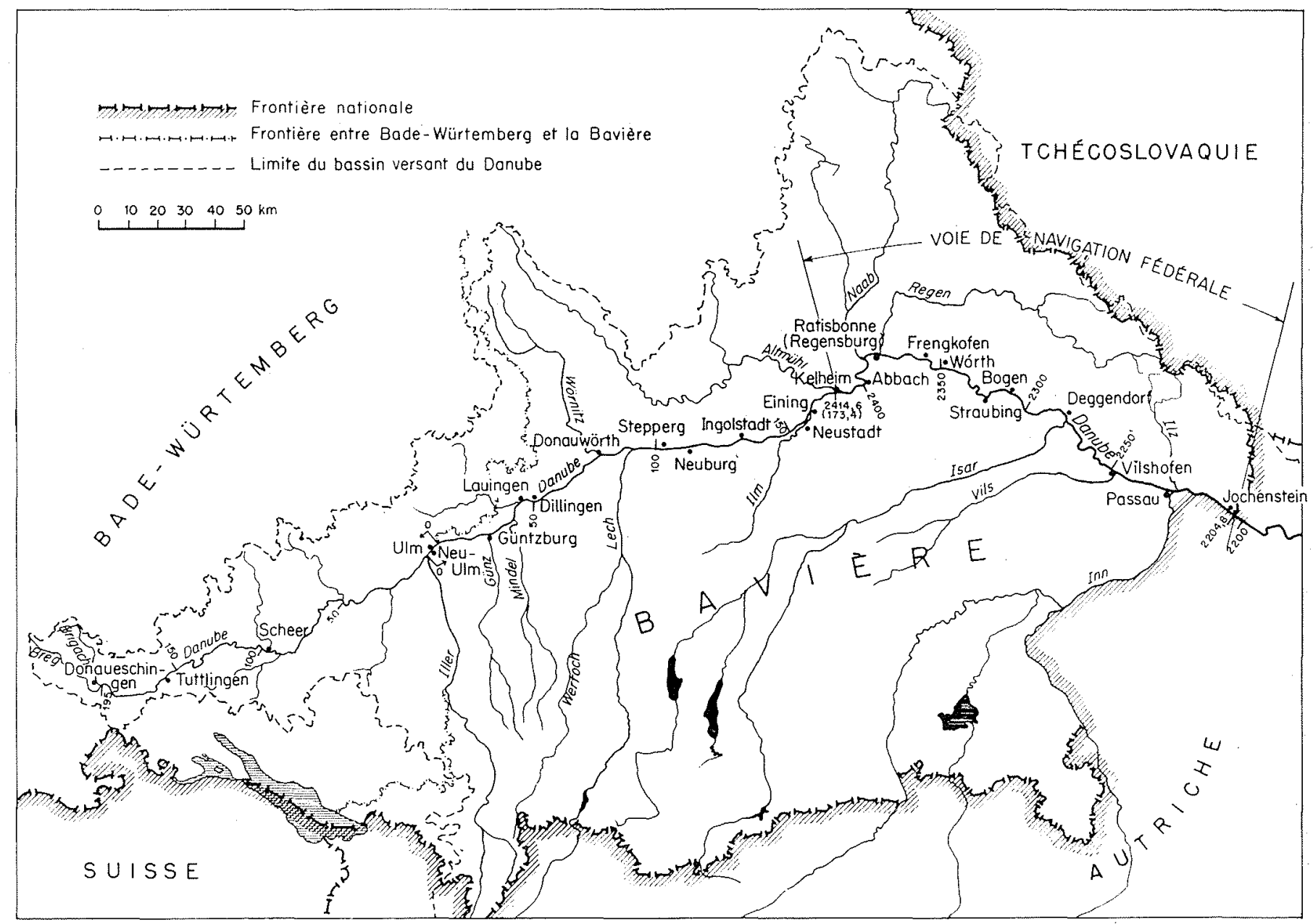

I/ Plan d'ensemble du Danube allemand, de Donaueschingen à Jochenstein.

Uebersichtslageplan der deastchen Donau von Donaueschingen bis Jochenstein. 
l'aval du débouché de l'Iller, à $23000 \mathrm{~km}^{2}$ à l'aval de celui de l'Altmühl à Kelheim. La pente diminue de $1,05 \%$ entre Neu-Ulm et Günzburg, à $0,43 \%$ à l'amont du confluent du Lech, se raidit ensuite et atteint $0,75 \%$ après quoi elle diminue de nouveau pour alteindre $0,32 \%$ à Kelheim. Les débits d'étiage minimal, de moyennes eaux et de crue annuelle sont, respectivement : $35 \mathrm{~m}^{3} / \mathrm{s}, 140 \mathrm{~m}^{3} / \mathrm{s}$, $350 \mathrm{~m}^{3} / \mathrm{s}$ à Neu-Ulm, et $90 \mathrm{~m}^{3} / \mathrm{s}, \quad 290 \mathrm{~m}^{3} / \mathrm{s}$, et $900 \mathrm{~m}^{3} / \mathrm{s}$ à Kelheim. Avant la construction des réservoirs d'accumulation dans les montagnes, les débits de crue les plus élevés à Kelheim pouvaient atteindre $2000 \mathrm{~m}^{3} / \mathrm{s}$.

Encore au début du $\mathrm{XIX}^{\circ}$ siècle, le Danube présentait toutes les caractéristiques des fleuves charriant des alluvions. Il s'écoulait par de multiples chenaux, variables après chaque crue, entre d'épais banes de gravier et les zones riveraines boisées d'étendue variable, provoquant de vastes submersions lors des grandes crues et causant des dégâts et des dévastations fort graves dans certaines localités particulièrement exposées à ces dangers.

Dès le début du $\mathrm{xIx}^{\circ}$ siècle, des organismes privés, des municipalités, et l'Administration Bavaroise elle-même, ont examiné les possibilités de protéger les zones exposées aux inondations par des ouvrages d'importance locale judicieusement disposés; le premier grand projet de régularisation a été mis en wuvre entre Lauingen et Dillingen pendant les années 1806 à 1814. Cependant, comme de telles mesures localisées, et séparées les unes des autres, ne pouvaient apporter une solution durable au problème, l'Etat Bavarois adopta dès les années 1830 des mesures envisageant l'aménagement et la stabilisation systématiques du lit du fleuve.

Une note de service officielle émise en 1834 précisait qu'il était essentiel, avant d'entreprendre des travaux de régularisation, de tout d'abord définir la largeur normalisée du chenal des moyennes eaux, compte tenu du fait que l'on cherchait, par la création d'un tel chenal stable et régularisé, à astreindre le fleuve à rester dans le même lit une fois pour toutes. Les largeurs normalisées choisies pour la régularisation des moyennes eaux ont été les suivantes :

- entre le débouché de l'Iller et celui du

Lech. . . ................

- entre le débouché du Lech et Ingolstadt.

$76 \mathrm{~m}$

- entre Ingolstadt et le débouché de

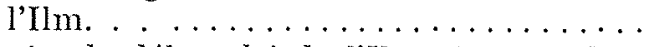

$95 \mathrm{~m}$

entre le débouché de l'Ilm et en aval de

$100 \mathrm{~m}$

Kelheim................... $117 \mathrm{~m}$

Des parties importantes du cours du Danube avaient été déjà aménagées dès 1850 , en fonction de ces normes, pour la protection des localités et des terres riveraines et pour l'amélioration de l'écoulement du fleuve. En 1862, une nouvelle loi bavaroise sur l'exploitation des eaux décréta que la correction systématique du fleuve serait désormais l'aflaire de l'Etat.

Les mesures essentielles adoptées pour cette correction du fleuve ont compris des coupures sur de longues distances, la construction d'ouvrages de fermeture des bras morts et d'anciens chenaux, et I'implantation de digues de guidage du courant afin de rétrécir la section d'écoulement du fleuve. Sur la partie bavaroise du cours, le choix s'est très vite porté sur des ouvrages en pierre, car il apparaissait que l'emploi de fascines ne pouvait offrir des garanties de sécurité suffisantes, étant donné les fortes variations du plan d'eau. Les mesures de correction prises à cette époque sur toute cette partie du cours ont, sans aucun doute, été exagérée en ce qui concernait la rectification $\mathrm{du}$ lit du fleuve.

La plus grande partie des travaux de correction du chenal des moyennes eaux entre UIm et Kelheim a été terminée au cours du $x x^{*}$ siècle, et il ne restait plus que quelques petits tronçons partiels à aménager pendant le siècle présent.

L'aménagement définitif de cette partie du Danube était terminé sur toute sa longueur de $173 \mathrm{~km}$, dès 1930 à la seule exception de quelque $3 \mathrm{~km}$ qui ne nécessitaient aucune mesure de correction.

La stabilisation du cours des eaux moyennes à l'intérieur d'un solide chenal n'est évidemment pas rester sans influencer le régime des crues dans toute la vallée fluviale. La régularisation du débit solide du fleuve, et l'établissement de conditions favorisant l'évacuation des glaces par le fleuve régularisé, ont certainement facilité l'écoulement des eaux de crue; par contre, les banes de matériaux de charriage déposés à certains endroits à la suite de la raideur parfois excessive de la pente du lit ont donné lieu à de nouveaux dangers. La régularisation du chenal des eaux moyennes est cependant restée sans effet sur les importantes superficies qui se trouvaient inondées lors de chaque grande crue, et c'est pour cette raison que des ouvrages de régularisation des crues (digues de protection) ont été construits, sur des longueurs importantes du cours, dès les premières années du $\mathrm{xrx}^{0}$ siècle.

Une digue de protection contre les crues a été construite sur la rive gauche du Danube en 1852 , s'étendant de Riedheim à Günzburg. Une autre a été construite en rive droite à Lauingen dès le début du $\mathrm{xrx}^{\circ}$ siècle, et s'étendait vers l'amont de cette localité. Les crues qui se sont produites à l'aval de Lauingen pendant les années 1894 à 1897 ont conduit à la réalisation d'un ensemble important de digues entre Dillingen et Donauwörth; ces ouvrages ont été construits par les diverses conmunes intéressées, et les travaux ont été subventionnés à la fois par le gouvernement local et par l'Etat. Cependant, toutes ces digues avaient été étudiées uniquement en fonction des crues estivales, et ne pouvaient, par conséquent, assurer une protection contre toutes les crues de l'année. A l'aval du confluent du Lech jusqu'à Ingolstadt, seules avaient été prises des mesures de protection contre les crues à des endroits isolés tels que Marxheim, Stepperg, Neuburg, Weichering, et à Ingolstadt même. Ce n'était qu'en aval d'Ingolstadt et jusqu'à Eining qu'on avait réalisé de plus importants enchaînements de digues pour la protection de la zone des basses terres fertiles et bien peuplées, qui atteignait par endroits une largeur de $4 \mathrm{~km}$.

A la suite d'une succession rapide, pendant les années 1920 , de fortes crues qui ont provoqué chaque fois la submersion de ces terres, l'Administration Bavaroise Suprême des Travaux Publics à Munich a résumé, dans un mémoire publié en 1927, toutes les mesures qui s'imposaient pour assurer une protection complète contre les eaux de crue dans ces secteurs. Il y était prévu, en particulier, la surélévation correspondante des digues d'été, la protection de toute la région fluviale située à l'amont du confluent du Lech (y compris le «Donauried»), ainsi que la surélévation des digues de protection situées à l'aval d'Ingolstadt, d'autant que le ren- 


\section{F. DOBMAYER.}

drait nécessaire la surélévation du plan d'eau provoquée par les dépôts de gravier qui se formaient à l'aval de cette localité. Le but recherché par ces aménagements était la protection d'environ 16000 ha de champs et de prairies, et de quelque 3000 ha d'îles et de forêts situés le long du parcours entre Neu-Ulm et Kelheim.

Ce projet d'aménagement complet et de construetion d'ouvrages complémentaires aux digues existantes n'a pourtant jamais été réalisé. Il a été abandonné parce que l'on ne concoit plus l'ensemble du problème des crues de la mềme manière qu'à cette époque-là. Le projet de construction de nouvelles centrales hydro-électriques à l'aval de la ville d'Ulm évite délibérément toute modification fondamentale du régime des crues.

Sur la partie du cours entre l'fller et le Lech, depuis les années 1930, on s'est simplement contenté de maintenir les digues existantes en bon état, et de ne construire que quelques ouvrages complémentaires. Quelques courtes digues de protection ont été réalisées entre le Lech et Kelheim, et dans les embouchures du Lech et de la Friedberger Ache, un aflluent de rive droite.

En 1946, une digue de fermeture longue d'environ $300 \mathrm{~m}$ a été achevée en rive gauche près de Bertholdsheim, et les localités de Dünzing et de Wackerstein ont été mises à l'abri des crues, de même que quelque 500 ha de terres agricoles et habitées au roisinage de Neustadt; les digues existant déjà près de Pförring ont été renforcées.

Le Danube à l'aval de la ville d'Ulm a toujours été le siège d'une navigation et d'un flottage. Déjà au XvıI ${ }^{\circ}$ siècle, les bateaux dits «Ordinari », pouvant transporter une charge de 10 à $20 \mathrm{t}$, assuraient un service régulier de transport de passagers entre UIm et Pressburg. Le transport des marchandises était assuré par les bateaux dits «Ulmer Schachteln» («Boîtes d'Ulm»), d'une charge utile de $150 \mathrm{t}$, qui servaient surtout au transport des matériaux de construction à destination de Vienne et de Pressburg. Une certaine période du $\mathrm{XrX}^{*}$ siècle a même vu l'exploitation d'une navigation à vapeur sur cette partie du cours, d'ahord vers l'aval à partir d'Ulm, et ensuite seulement à partir de Donauwörth. Mais les chemins de fer, et notamment l'ouverture de la ligne de la vallée du Danube en 1874 , ont porté un coup mortel à cette navigation fluviale à l'amont de Regensburg, et son importance n'a fait que diminuer à cause des performances limitées des bateaux dont on disposait à l'époque. La dernière «Ulmer Schachtel Danube en 1897, et à l'exception de quelques voyages occasionnels, toute navigation régulière a cessé entre Ulm et Kelheim à partir de ce moment. Aujourd'hui, seuls subsistent les bateaux d'excursions entre Kelheim et Weltenburg, qui restent toujours très appréciés du public. Le flottage ne se pratique plus depuis longtemps.

Il n'a pas été réalisé d'aménagements spéciaux pour la navigation autres que, d'une part, la régularisation du chenal des moyennes eaux (en stabilisant le fleuve à l'intérieur d'un chenal bien défini, on assurait, en même temps, des conditions plus favorables pour la batellerie) et d'autre part, quelques mesures exceptionnelles, telles que la correction du fleuve effectuée en 1842-1843 au pied du mont de Böfingen (à l'aval de la ville d'Ulm), et une régularisation expérimentale du chenal d'étiage près de Günzburğ.

\section{3. \\ Le Damube entre Kelheim et la frontière autrichienne à Jochenstein}

L'ensemble du parcours entre Kelheim et Jochenstein (long d'environ $213 \mathrm{~km}$ ) est situé en territoire bavarois, et jusqu'à l'année 1921, l'Administration bavaroise des Travaux Publics se chargeait de tous les aménagements intéressant la régularisation du lit des moyennes eaux, la protection contre les crues, et la navigation. Mais par la Convention d'Etat de 1921 sur le transfert de l'administration des cours d'eau navigables des « Länder» à l'Etat allemand, ce dernier est devenu responsable du parcours du Danube s'étendant du Kelheim juscu'à la frontière. L'Etat s'est engagé à prendre note de tous les problèmes intéressant la navigation, mais l'Administration bavaroise s'occupera, comme par le passé, du règlement des questions en dehors du chenal des moyennes eaux, c'est-à-dire, notamment, de la protection contre les crues et de la responsabilité des ports sur son propre territoire. Pour commencer, l'Etat allemand a eu recours aux services de l'Administration bavaroise pour l'exécution de ses propres tâches, mais il créa, d'abord provisoirement pendant la deuxième guerre mondiale, et ensuite définitivement le $1^{\mathrm{er}}$ avril 1947 , des services propres chargés des problèmes du Danube, et soumis au ministère des Transports de la République Fédérale.

En 1921, un autre événement important fut la signature, le 13 juin, d'une convention entre la Bavière et l'Etat allemand, portant sur la réalisation de la voie de navigation « Rhin-Main-Danube», et la création de la société "Rhein-Main-Donau A.G.». L'Etat allemand et les pays de Bavière et Bade confièrent à cette société, par la signature d'un contrat de construction, et d'un contrat de concession approprié, la réalisation de la grande voie de navigation «Rhin-Main-Danube» sur le parcours du Danube s'étendant d'Aschaffenburg jusqu'à Passau, et elle se chargea d'aménager cette voie fluviale pour permettre le passage de bateaux pouvant transporter des charges de 1200 à $1500 \mathrm{t}$.

Enfin, la convention définitive sur le Danube signée le 26 juin 1921 soumettait le parcours à l'autorité d'une commission internationale, le fleuve ayant été défini en tant que fleuve international par l'article 331 du Traité de Versailles.

Les principales caractéristiques géographiques et hydrologiques du Danube entre la frontière autrichienne et Jochenstein se résument succinctement comme suit :

Entre Kelheim et Regensburg, le Danube traverse encore les derniers contreforts du Jura franconien; il atteint le point le plus septentrional de son cours à Regensburg, s'infléchit vers le sud-est, et maintient ensuite cette dernière orientation sur l'ensemble de son parcours autrichien. A l'aval de Regensburg, le fleuve s'écoule par la riche plaine du Danube, qui constitue la limite nord-est du haut plateau souabo-bavarois. En rive gauche, le fleuve longe le massif primitf de la Forêt bavaroise et l'entame à plusieurs endroits; enfin, il en perce les contreforts et sort par une vallée étroite entre Hofkirchen et la frontière. On remarque la présence d'éperons rocheux à différents endroits dans le lit presque entièrement sablonneux et gravillonneux 


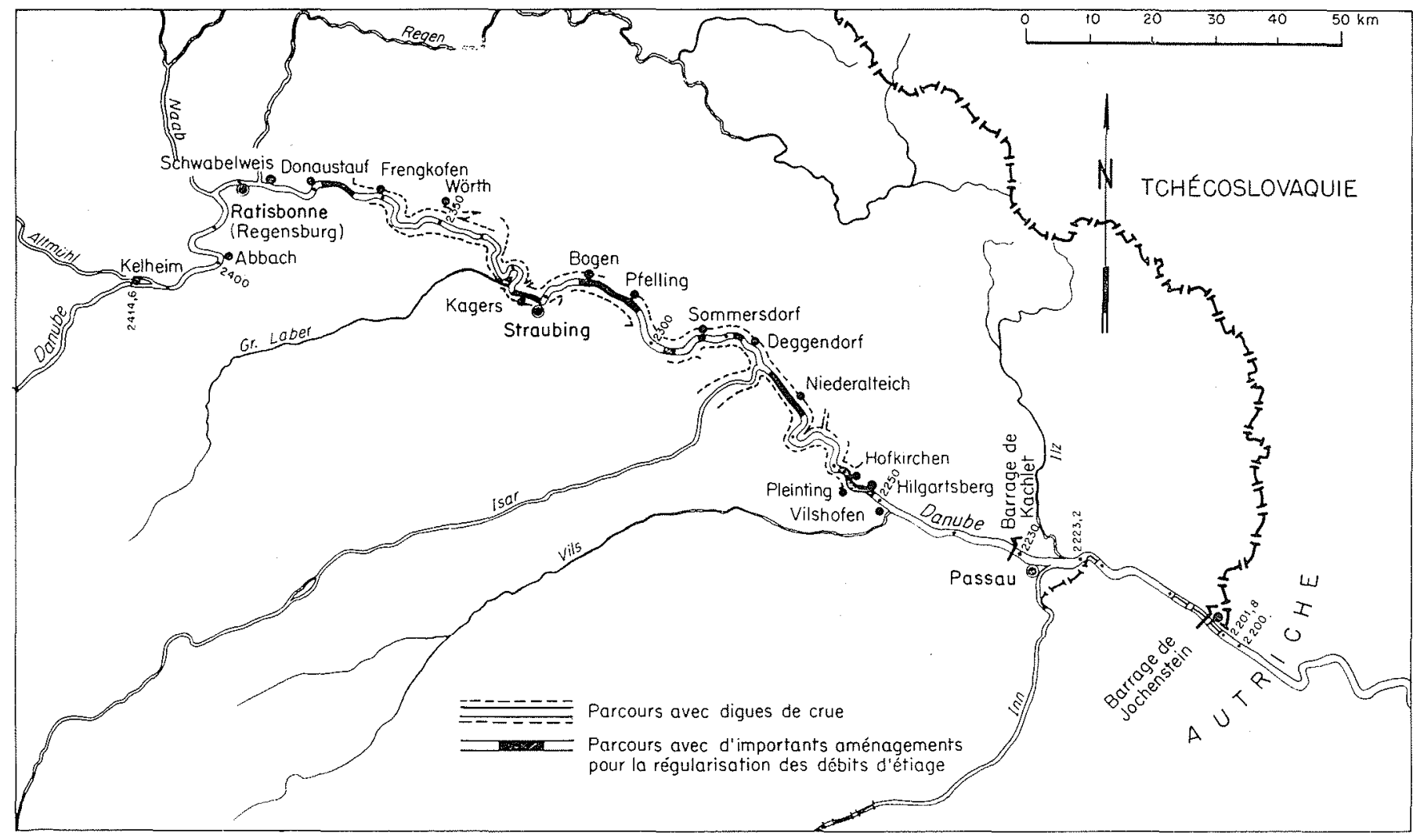

2/ Plan d'ensemble du Danube de Kelheim á Jochenstein (voie de navigation fédérale), avec digues de protection contre les crues et les plus importants ouvrages de régularisation du débit d'étiage. Uebersichtslageplan der Donall von Kelheim bis Jochenstein (Bundeswasserstrasse) mit Hochwasserschutzdämmen und grösseren Bauten der Niedertvasserregulierung.

du fleuve; par contre, à partir de Hofkirchen, les sables et les graviers sont remplacés surtout par les granites et les gneiss de la Forêt bavaroise.

Sur cette partie de son cours, le Danube reçoit successivement les eaux du Naab et du Regen (tous deux, rivières de moyenne montagne), mais à partir de Deggendorf - et surtout de Passau — son xégime est marqué de manière déterminante par les affluents de montagne, riches en eau et charriant de grandes quantités de dépôts, l'Isar et l'Inn. La contribution de la partie montagneuse du bassin versant du Danube atteint $41 \%$ à l'aval de Passau, et, à partir de là, c'est l'Inn qui détermine, en tant que rivière typiquement de haute montagne, le régime d'écoulement du Danube.

La superficie du bassin versant du Danube atteint $35000 \mathrm{~km}^{2}$ à Regensburg, passe à $38000 \mathrm{~km}^{2}$ à laval de Deggendorf, et enfin, à $77000 \mathrm{~km} 2$ à la frontière autrichienne à Jochenstein.

La pente naturelle du fleuve qui, entre Kelheim et Regensburg, varie entre $0,36 \%$ et $0,32 \%$, s'adoucit à $0,07 \%$ avant Deggendorf, mais atteint jusqu'à $0,43 \%$ à l'aval du confluent avec l'Isar, et surtout t partir du débouché de l'Inn à l'aval de Passau. Les retenues de Kachlet et de l'usine de Jochenstein compensent la raideur des parcours rocheux à l'amont et à l'aval de Passau.

Les retenues de Kachlet et de l'usine de Jochenot de crue annuelle correspondent, respectivement, aux points les plus caractéristiques à Schwabelweis, et à l'aval de Passau :

Schwabelweis : $135 \mathrm{~m}^{3} / \mathrm{s} ; 420 \mathrm{~m}^{3} / \mathrm{s} ; 1100 \mathrm{~m}^{3} / \mathrm{s}$. A l'aval de Passau: $390 \mathrm{~m}^{3} / \mathrm{s} ; 1300 \mathrm{~m}^{3} / \mathrm{s}$; $3000 \mathrm{~m}^{3} / \mathrm{s}$.
Le débit de la crue catastrophique du mois de juillet 1956 s'est élevé à $9300 \mathrm{~m}^{3} / \mathrm{s}$ à l'aval de Passau.

De même que pour le cours amont, les premiers travaux de régularisation des moyennes eaux ont été entrepris ici dès le début du xrx ${ }^{e}$ siècle. Des lar' geurs normalisées avaient également été prescrites pour le chenal d'écoulement par l'Administration bavaroise des Travaux Publics. Elles étaient les suivantes entre :

- Kelheim et le confluent du Naab...... $117 \mathrm{~m}$

- le confluent du Naab et Regensburg. ... $124 \mathrm{~m}$

- Regensburg et Straubing. . . . . . . . $132 \mathrm{~m}$

- Straubing et le confluent de l'Isar.... $146 \mathrm{~m}$

- les confluents de l'Isar, et de l'Inn à

Passau. . ................ $175 \mathrm{~m}$

- Passau et la frontière autrichienne... . $232 \mathrm{~m}$

Etant donné les conséquences parfois défavorables des raidissements de pente excessifs qui avaient été effectués à l'amont de Kelheim, on a procédé plus prudemment lors de la réalisation des travaux d'aménagement correspondant à l'aval de Regensburg; un certain nombre de boucles plus ou moins importantes ont été laissées à l'état naturel, ce qui a toutefois entraîné certaines conséquences défavorables au point de vue de la section et de la pente du fleuve. Tous les ouvrages de guidage et de protection des berges ont été réalisés en enrochements.

Les mesures essentielles de correction du chenal des moyennes eaux ont été achevées au cours des premières décennies du $\mathrm{xx}^{\circ}$ siècle. Sur ce parcours 
long de $213 \mathrm{~km}$, l'état d'avancement de l'aménagement en 1921 était le suivant : $122 \mathrm{~km}$ étaient corrigés et en état d'entretien, $55 \mathrm{~km}$ étaient corrigés mais avaient besoin de travaux complémentaires, $6 \mathrm{~km}$ ne nécessitaient aucune mesure, et $30 \mathrm{~km}$ (soit environ $14 \%$ du parcours) restaient encore sans aucun aménagement.

Ces travaux ont été exécutés par l'Administration bavaroise des Travaux Publics, et, pour la plus grande partie, aux frais de l'Etat de Bavière. Le restant des aménagements à prévoir par la suite a été pris en charge, à partir de l'année 1921, pa: l'Etat allemand qui en a fait exécuter une grande partie dans le cadre des travaux de régularisation des basses eaux dont était chargée la « Rhein-MainDonau A.G. ».

Les riches terres agricoles de la large plaine du Danube se trouvaient exposées, autrefois, aux dévastations provoquées régulièrement par les crues du fleuve. Avant les années 1930, pendant lesquelles l'Administration bavaroise des Travaux publics s'est décidée à résoudre une fois pour toutes le problème des crues, les seuls endiguements continus plus ou moins importants construits à l'aval de Kelheim se trouvaient dans la zone située entre Deggendorf et Pleinting; à part ceux-ci, il n'existait que quelques digues d'été au voisinage de Frengkofen et de Wörth, à l'aval de Regensburg. Il ne s'agissait d'ailleurs que de digues d'été de faible hauteur, qui se trouvaient submergées par chaque crue plus ou moins importante. La protection de Bad Abbach a été entreprise pendant les années 1927 à 1929 , sur la base d'un projet d'aménagement général établi par l'Administration bavaroise des Travaux Publics en 1927, mais ces travaux n'ont pu être achevés qu'à la réalisation de l'ensemble des mesures de drainage des terres entre les années 1953 et 1957 .

La plupart des travaux d'aménagement de la plaine d'inondation entre Frengkofen et Wörth ont été menés à bien dès les années 1927-1931, mais ils n'ont pu être achevés dans l'ensemble qu'en 19521953 , lors du renforcement des digues de retenue et de l'implantation des ouvrages de drainage des terres. La plus importante de toutes les mesures prises contre les crues intéressait les trois bassins situés, respectivement, à Straubing, à Deggendorf, et à Pleinting. Les digues et les installations de drainage nécessaires ont été réalisées dans la zone de Deggendorf en 1926-1932, et dans celles de Straubing et de Pleinting en 1936-1941. Ces travaux n'ont toutefois pu être achevés qu'en 1957-1959.

Il avait été décidé avant tout de réaliser un endiguement complet, comprenant toutes les digues de retenue nécessaires sur les affluents, ainsi que l'ensemble des installations de drainage des terres. Les digues principales comportent une crête large de $2 \mathrm{~m}$ s'élevant à $0,80 \mathrm{~m}$ au-dessus de la cote des plus hautes eaux, des talus disposés à la pente de $1: 2$, et elles consistent essentiellement en un massif de gravier, sans noyau d'étanchéité spécial, et recouvert d'une couche de terre végétale ou de gazon. Leur longueur totale atteint $228 \mathrm{~km}$.

La restitution des eaux de drainage dans le Danube est assurée par 44 stations de pompage. La superficie totale ainsi protégée s'élève à environ 12000 ha, et l'ensemble de ces travaux d'aménagement a coûté 65 millions de Reichmark et DeutscheMarks, dépense dont la plus grande partie a été à la charge de l'Etat de Bavière. Il n'a été demandé aux communes intéressées par ces travaux de participer aux dépenses que dans la mesure de leurs possibilités.

Des ouvrages de protection contre les crues ont été construits dernièrement près de Demling, à l'aval de Regensburg. Grâce à la construction d'une route de contournement le long du Danube à Vilshofen, il a pu être assuré, à la fois une protection complète contre les crues, des bas-quartiers de cette ville situés sur la rive gauche de la Vils, et les installations de drainage nécessaires. Des travaux sont actuellement en cours pour résoudre le problème des crues menacant la ville de Passau, qui est plus exposée que toute autre localité à ce point de vue. On étudie actuellement, sur un modèle, des mesures de protection pour la ville de Regensburg; le projt définitif sera dressé sur la base des résultats de ces essais. L'Administration bavaroise des Travaux Publics attaque également le problème des crues du Danube d'une autre manière, en cherchant à les écrêter au moyen de grands réservoirs-tampons sur les affluents du fleuve, notamment ceux de Sylvenstein sur l'Isar, et de Forggensee, près de Rosshaupten dans la région du Lech.

Alors que pratiquement toute navigation à l'amont de Kelheim avait déjà disparu avant la fin du XIX siècle, il subsistait par contre encore à cette époque un trafic fluvial relativement intense entre Kelheim et Regensburg, avec des bateaux pouvant transporter des charges de l'ordre de $120 \mathrm{t}$, du moins tant que le canal «Ludwig » du Danube au Main restait encore accessible. Les matériaux de construction et les céréales étaient les principales marchandises transportées par ces bateaux. Par contre, depuis les quelques dernières décennies il ne subsiste plus qu'un trafic de passagers occasionnel sur cette partie du cours, ainsi que quelques transports de matériaux de construction pour les travaux d'entretien du ressort de l'Administration.

La situation est tout à fait différente sur le cours à l'aval de Regensburg, qui est parcouru depuis déjà longtemps par des chalands de 600 à $700 \mathrm{t}$, longs d'une soixantaine de mètres et de $8 \mathrm{~m}$ de largeur. La ville de Regensburg doit de toute manière être considérée comme marquant le point de départ de la navigation (proprement dite) pour de grands bateaux sur le Danube. C'est d'ailleurs justement parce qu'il s'était développé une navigation déjà appréciable dès le début du présent siècle - grâce, entre autres choses, à la réalisation des travaux de correction du chenal des moyennes eaux - que les autorités bavaroises ont été poussées à réaliser en temps utile des installations spécialement concues pour le trafic fluvial, parmi lescuelles notamment:

- les quais construits à Regensburg pendant les années 1835-1906, dont la longueur totale est de l'ordre de $2700 \mathrm{~m}$;

- le port «Luitpold» à Regensburg, comportant deux bassins, dont l'un pour le transbordement des marchandises, et l'autre pour les produits pétroliers. Ces installations remontent aux années 1908-1911;

- le port de refuge à Deggendorf, qui fut construit pendant les années 1898-1908;

- les installations d'appontement (embarcadères), et un quai long de $760 \mathrm{~m}$ à Passau, construits entre 1890 et 1894 ;

- le port d'hiver et de transbordement de Passau, 


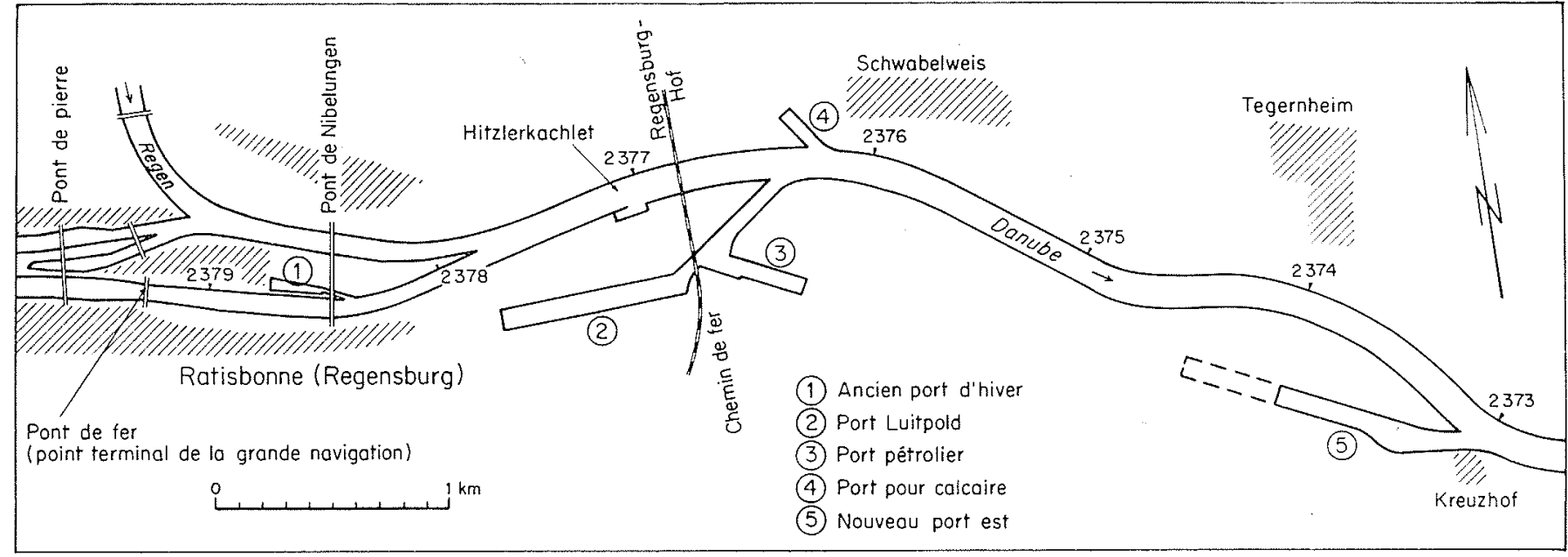

3/ Les installations du, port de Regensburg.

Hafenanlagen in Regensburg.

avec un quai de $700 \mathrm{~m}$ de long, construit pendant les années 1899-1902;

- l'embarcadère à Obernzell.

L'amélioration du chenal de navigation dans la partie du cours dite des « Kachlets $\gg$ entre Vilshofen et Passau sur une longueur d'environ $30 \mathrm{~km}$ est igalement digne d'intérêt; en l'approfondissant à l'aide d'explosifs (les premiers travaux de ce genre y furent exécutés en 1820) on a cherché à assurer un tirant d'eau d'au moins $1,40 \mathrm{~m}$ sur une largeur de $40 \mathrm{~m}$. Cette partie du cours était la plus dangereuse sur l'ensemble du Danube allemand; les "Kachlets», parties du cours à fond rocheux, constituent une menace pour la navigation. Ce sont eux, d'ailleurs, qui ont donné leur nom au barrage de Kachlet, dont une description plus détaillée figure plus loin.

Des études avaient déjà été faites avant la fin de la première guerre mondiale, afin de rechercher une solution qui permettrait de régulariser le chenal d'étiage sur l'ensemble du parcours de Regensburg à Passau et, en augmentant les profondeurs d'eau disponibles, de répondre aux besoins de la navigation et d'en améliorer les conditions. Ces etudes devaient également assurer les bases des programmes de création de travail prévus pour la période d'après-guerre. Le Service de Construction des Canaux, mis sur pied par l'Administration bavaroise des Travaux Publics pour la réalisation de la grande voie de navigation Aschaffenburg-Passau, ¿ établi en 1918 un projet, visant l'amélioration, par la régularisation du chenal d'étiage, des conditions de navigation dans les parcours présentant des fonds meubles constitués de graviers à l'aval de Regensburg. L'Inspectorat des Canaux à Regensburg a ensuite mis au point, toujours pendant la même année 1918, un projet de régularisation du chenal d'étiage, prévoyant l'écoulement de ces débits en suivant étroitement le thalweg créé par les mesures de correction du chenal des moyennes eaux, l'adoucissement des courbes particulièrement serrées, et la création d'une profondeur d'eau de $2 \mathrm{~m}$ lors de la plus basse cote du plan d'eau navigable. On cherchait à alteindre ces objectifs essentiellement au moyen de coupures isolées, d'adoucissement des courbes, et par la mise en place d'ou- vrages de guidage, d'épis transversaux, et de seuils de fond. Mais, étant donné, d'une part, que l'on disposait à l'époque d'une expérience encore insuffisante quant au choix des meilleurs profils pour les épis et les ouvrages longitudinaux, et d'autre part, que l'on désirait pouvoir se faire une idée de l'effet de ces ouvrages, on a commencé par aménager, en 1920, d'assez longs troncons expérimentaux à Niederalteich $(\mathrm{km} 2276)$ à l'aval du confluent de l'Isar, et à Donaustauf ( $k m$ 2369) à l'aval de Regensburg.

Ces travaux réalisés uniquement dans l'intérêt de la navigation, avaient été projetés et mis en œuvre, juscju'à ce moment-là, par l'Administration bavaroise des Travaux Publics.

Par la signature de la convention d'Etat de 1921, à laquelle il a déjà été fait allusion plus haut, la responsabilité de l'entretien et de l'exploitation du chenal navigable (sauf pour les ponts et appontements à Regensburg et à Passau) est passée à l'Administration Nationale des Voies Navigables. Les nouveaux travaux de régularisation du chenal d'étiage ont été entrepris par la « Rhein-MainDonau A.G. », créée en 1921.

Les hauteurs d'eau disponibles pour la navigation aux plus mauvais passages entre Regensburg et Passau atteignaient à cette époque : $14 \mathrm{dm}$ à Donaustauf à l'aval de Regensburog, $15 \mathrm{dm}$ à l'aval de l'embouchure de l'Isar, 13 à $14 \mathrm{dm}$ dans les "Kachlets» à l'amont de Passau, et $17 \mathrm{dm}$ à l'aval de cette ville.

Des travaux essentiels ont été entrepris pendant les années 1920 et 1930 pour améliorer les conditions de navigation. Pendant les années 1922-1927, la «Rhein-Main-Donau A.G. » a entrepris, sur la base de projets antérieurs, la construction du barrage et de l'usine de Kachlet à l'amont de Passau. Cet aménagement au fil de l'eau, l'un des plus puissants de son genre en Europe à l'époque, comportait huit turbines Francis avec une puissance installée de $42000 \mathrm{~kW}$, et une productivité annuelle de $275000000 \mathrm{kWh}$. Le barrage comporte six passes de $25 \mathrm{~m}$ de large, avec volets déversants. L'écluse, du type double, a une longueur utile de $230 \mathrm{~m}$, et une largeur utile de $24 \mathrm{~m}$. La chute s'élève à $9 \mathrm{~m}$ en régime d'étiage, et varie considérablement, 


\section{F. DOBMAYER.}

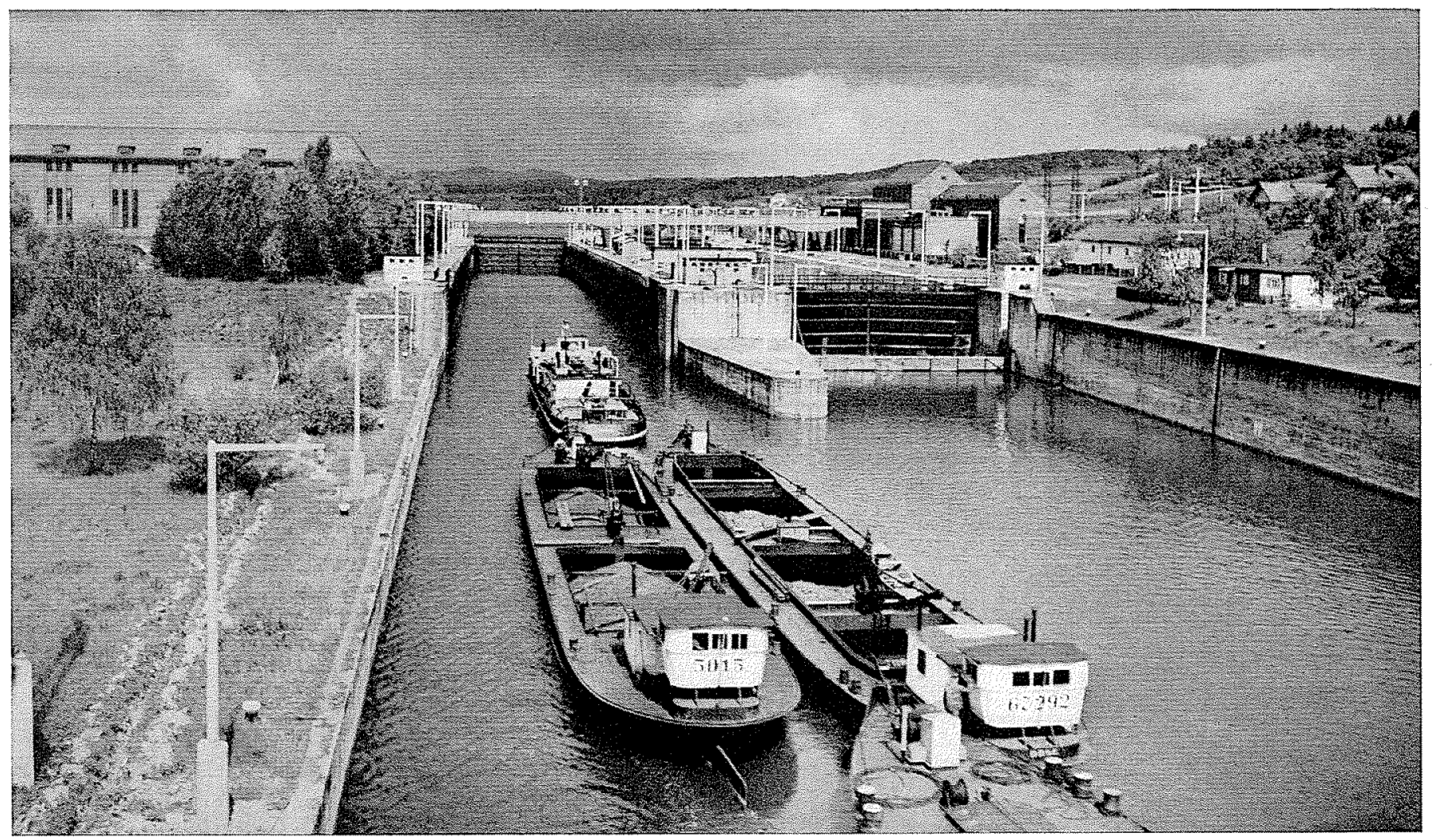

Q/ L'écluse de Kachlet. Vue d'aval.

Schleuse Kachlet von Unterstrom gesehen.

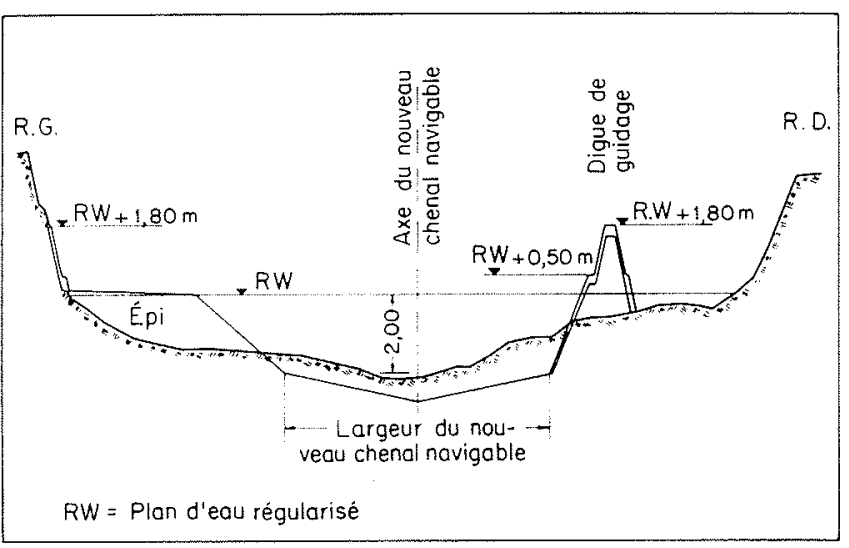

5/ Régularisation d'étiage. Profil type du fleuve.

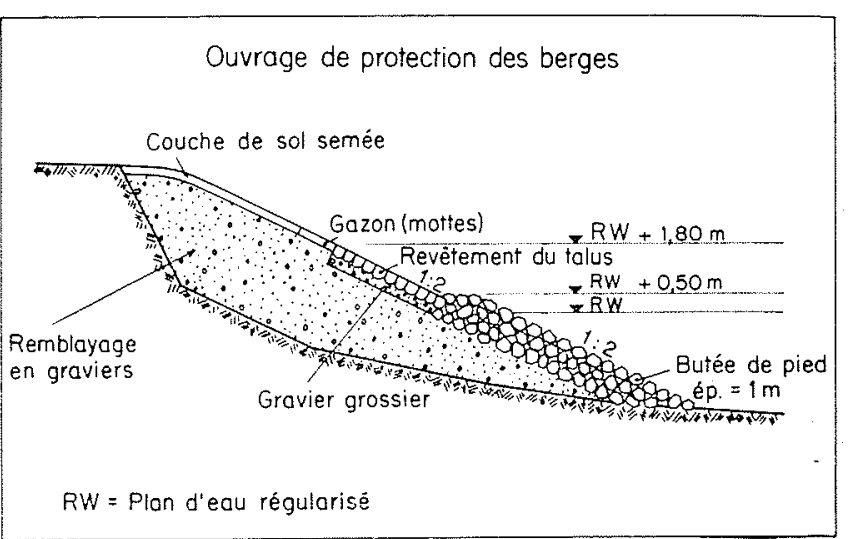

6/ Régularisation d'étiage. Profil type d'un ouvrage de protection des berges.

Niederwasserregulierung, Regelquerschnitt eines Uferdeckwerkes. schnitt.

charakteristischer Flussquer-

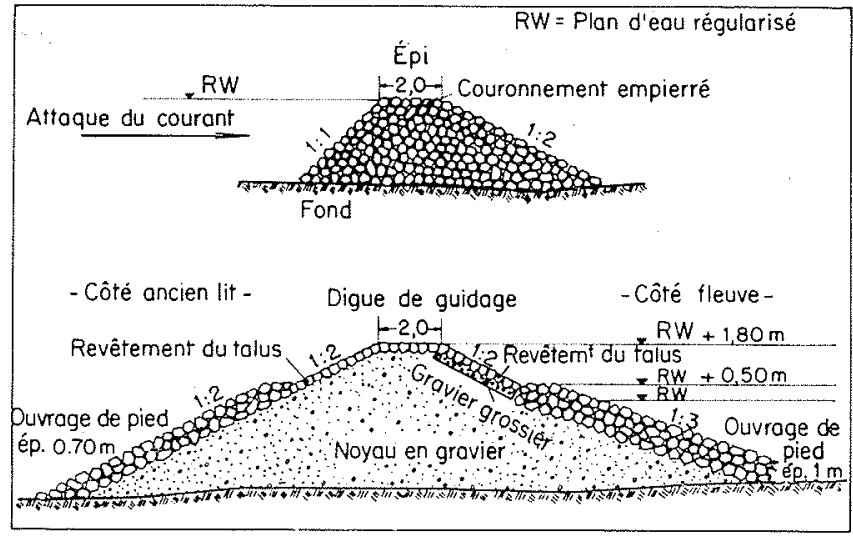

7/ Régularisation d'étiage.

Profil type d'un épi et d'un ouvrage-guideau.

Niederivasserregulierung.

Regelquerschnitt einer Buhne und eines Leitwerkes. 
suivant les différents débits et cotes du plan d'eau uval. La largeur de l'écluse a été choisie de telle vorte que même les bateaux à roues à aubes, très larges, puissent y passer sans risque. La Commission Internationale du Danube est intervenue lors du choix des dimensions de cette écluse, et a laissé ontendre, par ailleurs (comme elle l'a fait également pour la nouvelle écluse de Jochenstein) qu'aucune faxe ne devait ètre perçue sur la navigation empruntant cette écluse. La seule condition détermiinante à intervenir dans le choix de la cote de retenue a été l'altitude de la ville de Vilshofen, siluée à une vingtaine de kilomètres à l'amont du barrage. La retenue n'atteint pas la partie rocheuse Au cours dite «Hilgartsberger Kachlet», qui sétend en amont sur une dizaine de kilomètres.

Diverses mesures de régularisation des débits d'étiage ont également été entreprises au cours de ces mèmes années, tout d'abord, bien entendu, aux cndroits où la navigation éprouvait de grandes difficultés par suite d'une profondeur d'eau insuffisante (dans les surlargeurs), ou par suite de brusques ruptures de pente, ou bien dans les courbes excessivement serrées. De nombreux épis ont été implantés à divers endroits pendant les années 1920, notamment à l'aval du confluent de l'Isar $(\mathrm{km} 2280)$, afin de fixer une fois pour toutes la position du chenal d'écoulement, très variable à cel endroit.

Ces mesures n'ont cependant pas apporté une solution définitive au problème, car le Danube ne peut de lui-même évacuer qu'une certaine partie des matériaux que l'Isar lui apporte chaque année, bien que le volume de ces apports soit actuellement en voie de diminution d'une année à l'autre. Malgré les améliorations successives apportées à cette partie du fleuve, sa régularisation définitive reste toujours inachevée, et ne sera sans doute parfaite qu'au moment où, grâce à la réalisation de la dernière étape d'aménagement hydroélectrique de l'Isar, cette rivière ne charriera plus de matériaux, de sorte que les pentes de son dernier troncon aval pourront se stabiliser au bout d'un certain temps. L'aménagement d'épis à Metten $(\mathrm{km} 2288)$ et à Kleinschwarzach $(\mathrm{km} \mathrm{2292)}$, à l'amont de Deggendorf, a réduit efficacement des surlargeurs qui existaient à ces endroits.

L’aménagement du cours a été particulièrement difficile à Kagers, à l'ámont de Straubing; c'est sur ce parcours, près du lieudit «Wundermühle», au $\mathrm{km}$ 2323, que se trouvait la courbe la plus serrée du Danube allemand, dont le rayon de $180 \mathrm{~m}$ a été porté à $300 \mathrm{~m}$ par la suite. Cette mesure n'a cependant pas entièrement réussi à assurer les conditions voulues, étant donné la nécessité de construire très économiquement, compte tenu de l'insuffisance des moyens disponibles pour la construction. Une correction de petite envergure du chenal d'étiage avait également été entreprise à cette époque à Schildorf, à l'aval de Passau, dans une zone à présent englobée dans la retenue de l'usine de Jochenstein. Des travaux de correction plus ou moins importants ont été exécutés à seize endroits différents, assurant une profondeur d'eau navigable de $2 \mathrm{~m}$ sur $37 \mathrm{~km}$ des $130 \mathrm{~km} \mathrm{du}$ parcours entre Regensburg et Vilshofen. Quelque cent cinquante épis ont été implantés, ainsi que cinq seuils de fond, des ouvrages de guidage d'une longueur totale de $6000 \mathrm{~m}$, et des ouvrages de protection des berges s'étendant sur environ $5000 \mathrm{~m}$ nécessitant la mise en cuvre de $150000 \mathrm{~m}^{3}$ d'enrochements, et d'environ $1300000 \mathrm{~m}^{3}$ de gravier. Après la construction de la centrale de Kachlet, et la régularisation des passages les moins profonds, la profondeur d'eau à la disposition de la navigation atteignait, d'après les calculs effectués à l'époque, $1,60 \mathrm{~m}$ pendant 332 jours d'une année moyenne, alors qu'en 1918, cette profondeur n'avait pu être assurée que pendant 306 jours. La navigation fluviale comportait a l'époque surtout des chalands de $70 \mathrm{~m}$ jaugeant $1000 \mathrm{t}$. En raison de leur plus grande longueur, ces derniers n'ont pu naviguer sans difficulté qu'après l'achèvement de ces mesures de régularisation.

Un nouvel embarcadère et une installation de transbordement ont été construits pendant les années 1930 par un organisme privé à l'aval de Deggendorf, et en 1935, l'Administration Nationale des Voies de Navigation Fluviales a construit un petit port de refuge à Lindau, à l'aval de Passau, pour servir de garage pour les bateaux-citernes.

La loi sur la liaison Rhin-Main-Danube et l'aménagement du Danube prévoyant lachèvement de la grande voie de navigation du Rhin au Danube par le Rhin au plus tard en 1945, est entrée en vigueur en 1938 , et semblait devoir provoquer un renouvellement des efforts en vue d'assurer la régularisation efficace du fleuve. Un nouveau projet définitif de régularisation du chenal d'étiage entre Vilshofen et Regensburg a été établi en 1938, sur la base des projets qui avaient été étudiés en 1918-1921, et compte tenu de l'expérience acquise au cours des travaux de correction réalisés depuis ces années. Ce projet prévoyait l'établissement d'une profondeur d'eau navigable d'au moins $2 \mathrm{~m}$ avec des largeurs de chenal suffisantes, et des rayons toujours égaux ou supérieurs à $500 \mathrm{~m}$ pour les courbes. Les calculs hydrauliques effectués ont donné des largeur's de chenal de navigation s'échelonnant entre 70 et $120 \mathrm{~m}$ selon les pentes des divers tronçons aménagés. De même que les études effectuées antérieurement, ce projet se basait sur un débit aménagé de $210 \mathrm{~m}^{3} / \mathrm{s}$ au droit du limnimètre de Regensburg-Schwabelweis, pour la partie du cours entre cette localité et le confluent avec l'Isar, auquel correspond, d'après les observations recueillies actuellement au limnimètre de Hofkirchen pour le tronçon entre l'Isar et Passau, un débit de $338 \mathrm{~m}^{3} / \mathrm{s}$, avec la même durée de dépassement pour ces deux valeurs. Le projet, prévoyant des épis et des seuils de fond réalisés entièrement en enrochements, et des ouvrages de guidage et de protection comportant des noyaux de gravier, envisageait un aménagement assez poussé de l'ensemble du parcours, afin d'assurer une bonne marge de sécurité économique. Les prévisions portaient sur la mise en œuvre de $800000 \mathrm{~m}^{3}$ d'enrochements et de 6000000 de $m^{3}$ de graviers, ces derniers provenant de travaux à la drague flottante, et étant réemployés partiellement dans les ouvrages. Mais les seuls et derniers travaux réalisés pendant les premières années de 1940 ont porté sur la construction de quelques ourrages de guidage à Wischlburg (km 2299), et à Hermannsdorf (km 2309).

Le trafic de transbordement des marchandises a atteint en 1938 un premier chifre record de $1300000 \mathrm{t}$ à Regensburg (alors que ce port était concu pour 1000000 de tonnes seulement), mais 
il a cessé entièrement vers la fin de la deuxième guerre mondiale, de même que tous les travaux de régularisation et d'aménagement du fleuve. Il a cependant repris rapidement à partir de 1948 , et a fini par largement dépasser son importance d'avant-guerre. Le chiffre de 1300000 a été atteint de nouveau dès 1951, puis dépassé en 1952 avec $2300000 \mathrm{t}$. Le record d'après-guerre a été atteint en 1957, avec $3100000 t$ de marchandises transbordées. Le trafic fluvial a tout d'abord repris avec l'Autriche et, depuis ces dernières années, avec tous les autres pays du Danube, de sorte que l'on retrouve aujourd'hui des bateaux provenant de tous ces pays dans le port de Regensburg. Cette étonnante croissance de la navigation a inévitablement rendu nécessaire de nouvelles mesures pour l'amélioration du cours du Danube allemand qui, en raison de ses caractéristiques naturelles débits relativement peu importants, lit mineur étroit, successions de courbes à faible rayon), constitue la partie la plus défavorable de l'ensemble du fleuve entre Regensburg et Sulina.

Des efforts importants ont été faits à partir de 1949 pour achever les travaux de régularisation du chenal d'étiage dans les plus brefs délais. Outre de nombreuses mesures secondaires, on a réalisé les importants aménagements suivants :

- Le tracé du chenal d'écoulement et la transition amont ont été améliorés à la courbe dite «Rainer Eck» située à l'amont de Hofkirchen

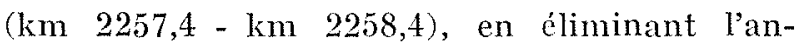
cienne courbe, à faible rayon et difficilement navigable, au moyen de travaux de dragage, et par la construction d'un ouvrage de guidage et de protection sur une longueur d'environ $1000 \mathrm{~m}$ suivant la rive droite du chenal;

- Le chenal de navigation à voie unique et mal disposé à l'amont de Deggendorf a été déplacé du bras gauche (du fleuve) vers la droite, à côté de l'île de Sommersdorf, et sa largeur a été portée à une centaine de mètres. Le bras gauche du fleuve a ensuite été fermé au moyen d'un mur-guideau long de $1,3 \mathrm{~km}$, s'étendant du $\mathrm{km} \mathrm{2293,1}$ au $\mathrm{km} 2294,4$ en rive gauche, et jusqu'au-delà du côté droit de l'île. Il a été nécessaire, en outre de draguer un volume de gravier d'environ $100000 \mathrm{~m}^{3}$ dans le bras droit du fleuve;

- Dans le troncon à faible pente entre le $\mathrm{km} \mathrm{2296,0}$ et le $\mathrm{km} 2298,5$ à Mariaposching, on a, par l'aménagement d'ouvrages de guidage, de nombreux épis très longs, et d'importants travaux à la drague, fixé le chenal de navigation sur une distance de plus de $2 \mathrm{~km}$, de manière à $\mathrm{y}$ assurer une profondeur d'eau de $2 \mathrm{~m}$ sur une largeur d'au moins $100 \mathrm{~m}$;

- Le thalweg du fleuve a été fixé sur le parcours, long d'environ $7 \mathrm{~km}$, entre Pfelling et Sand (km 2305,0 - km 2312,5) par l'aménagement d'épis et de digues de guidage; on a cherché, en améliorant le tracé hydraulique du chenal d'écoulement, à éviter les embâcles très fréquents qui se produisaient dans cette partie du cours, et en fait, aucun embâcle ne s'y est produit depuis cet aménagement;

- Dans le parcours rochenx dit «Hilgartsberger Kachlet $\gg$, entre le $\mathrm{km} 2251,0$ et le $\mathrm{km} 2259,0$ (qui est hors de lemprise de la retenue de l'usine de Kachlet), on entreprend depuis plusieurs années d'importants travaux à la drague et aux explosifs, dans le but d'aménager dans ce parcours un chenal de navigation profond de $2 \mathrm{~m}$. Quelque $40000 \mathrm{~m}^{3}$ de rocher et $10000 \mathrm{~m}^{3}$ de gravier auront été enlevés du lit à l'achèvement de ces travaux.

On envisage par ailleurs, et en fonction des résultats d'essais sur modèle actuellement en cours du fleuve à Kagers, en amont de Straubing (auquel il a déjà été fait allusion plus haut) en exécutant des travaux plus importants ayant pour objet une meilleure adaptation entre elles des courbes à faible rayon se suivant de près sur ce parcours.

La société « Rhein-Main Donau A.G.» doit, en principe, terminer les travaux de régularisation du chenal d'étiage sur le parcours Regensburg-Vilshofen, et remettre cette réalisation à l'Administration Fédérale des Eaux et de la Navigation dès 1965.

Le coût global des travaux de régularisation des débits d'étiage aura atteint, à ce moment-là, la somme de 40 à 50 millions de DM (en valeur d'aujourd'hui).

Après l'aménagement du fleuve entre Vilshofen et Regensburg, les conditions de navigation s'y présenteront comme suit :

- Pour un plan d'eau à la cote de $116 \mathrm{~cm}$ au droit de Schwabelweis, on peut prévoir qu'une profondeur d'eau navigable de $2 \mathrm{~m}$ sera disponible sur l'ensemble de ce parcours pendant 308 jours par an (prévision basée sur la moyenne de plusieurs années), déduction faite des $7+14=21$ jours de crues et de glace interdisant la navigation pendant une année moyenne. Or, actuellement, cette profondeur de $2 \mathrm{~m}$ n'est disponible que pendant 251 jours de l'année.

En 1918, c'est-à-dire avant la réalisátion des premiers travaux, la profondeur de $2 \mathrm{~m}$ était disponible pendant 217 jours seulement.

Si l'un des buts du projet initial (c'est-à-dire l'aménagement d'un chenal de navigation présentant une profondeur d'eau garantie de $2 \mathrm{~m}$ ), a été atteint grâce à ces travaux de régularisation, ceux-ci ne permettront pas, par contre, d'assurer les largeurs de 70 à $120 \mathrm{~m}$ prévus pour ce chenal. La raison essentielle en est que le fleuve s'approfondit de $1-2 \mathrm{~cm}$ chaque année (surtout à l'aval de Regensburg) et que cet approfondissement réduit la largeur de la partie active de chaque section d'écoulement.

On disposera, à l'étiage navigable, après aménagement, des largeurs de chenal de navigation suivantes dans les diverses parties du cours intéressé;

- entre Vilshofen et Straubing : au moins $70 \mathrm{~m}$ dans les troncons rectilignes, avec des surlargeurs correspondantes dans les courbes, ce qui assurera un chenal navigable à deux voies, sauf dans la courbe à faible rayon dite "Mühlhammer Kurve», dans la zone à l'aval du confluent de l'Isar, et dans un court troncon. à l'aval de Straubing;

- à l'amont de Straubing : $40 \mathrm{~m}$ dans les troncons rectilignes, et surlargeurs correspondantes dans les courbes, ce que permettra seulement une navigation à voie unique sur pratiquement l'ensemble de ce parcours. Un nombre adéquat de 


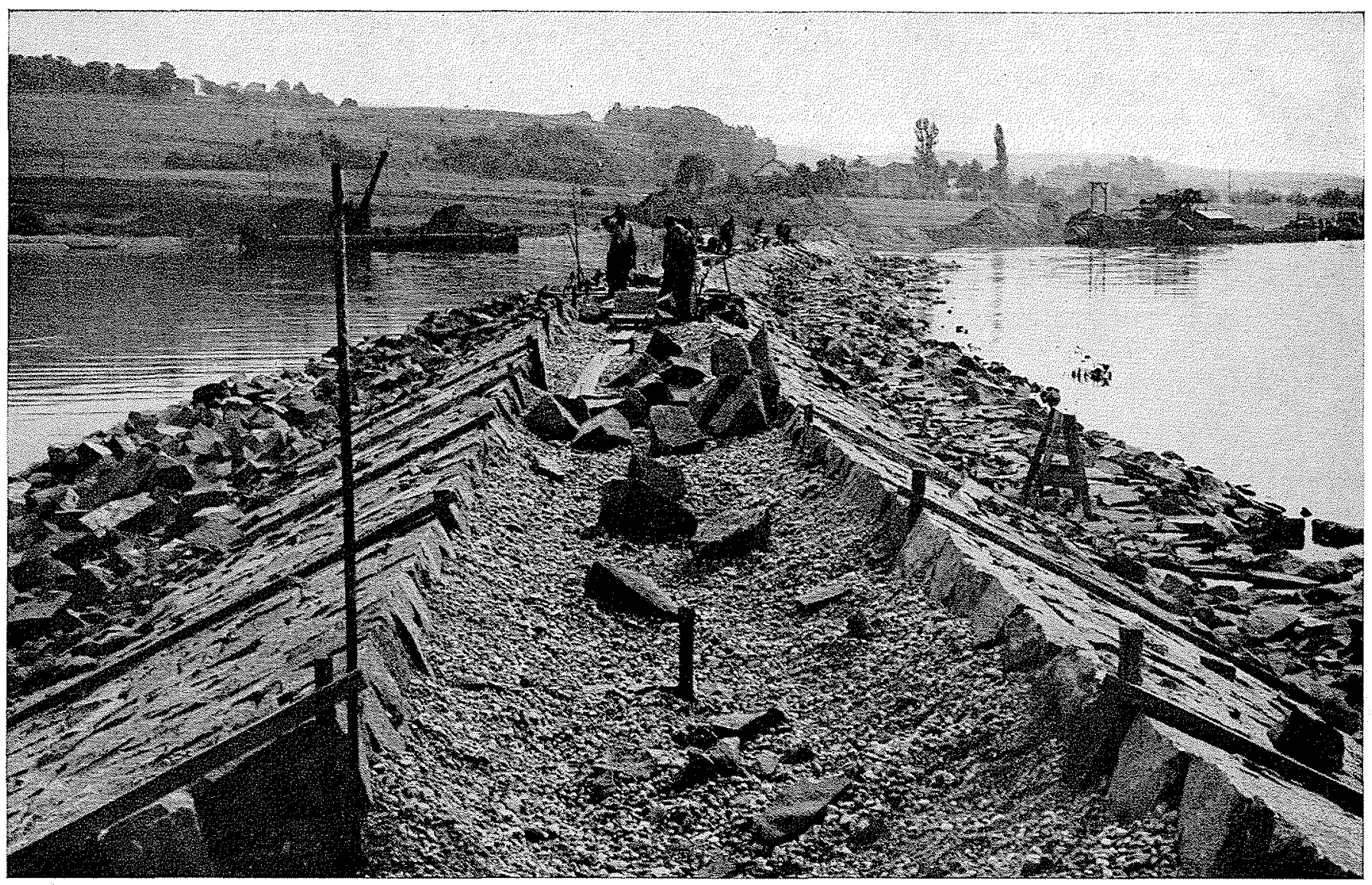

8/ Régularisation d'étiage. Construction d'un ouvrage-guideau en rive gauche, au « Rainereck», $\mathrm{km} 2258$. Niederwasserregulierung, Baumassnahme «Rainereck», $\mathrm{km} 2258$, Ball des Leitwerks am linken Ufer.

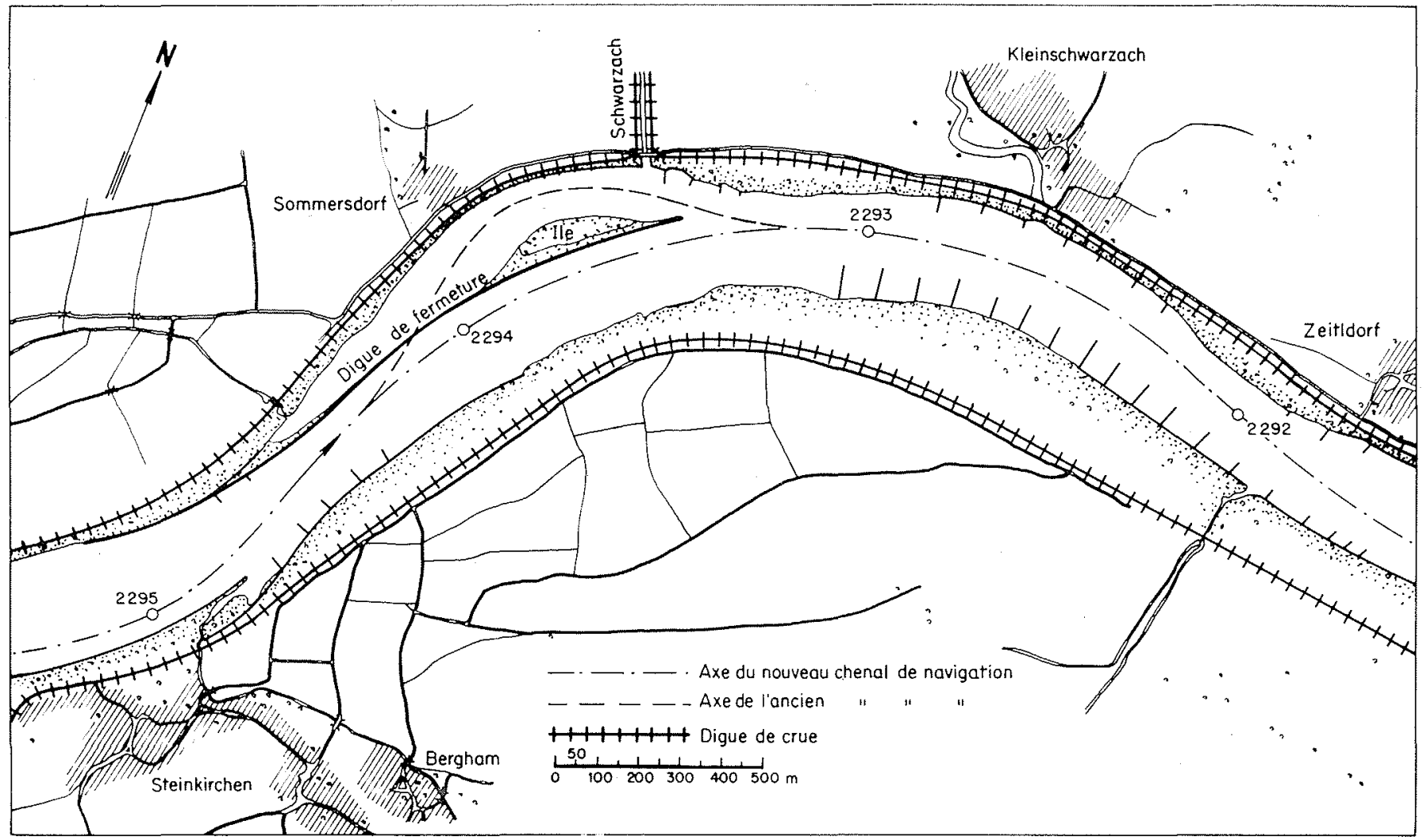

9/ Régularisation d'étiage. Plan de situation des ouvrages à Sommersdorf, km 2294. Niederwasserregulierung, Lageplan der Baumassnahme Sommersdorf, km 2294. 


\section{F. DOBMAYER.}

garages de croisement uniformément répartis sera prévu, et les bateaux devront s'arranger entre eux par T.S.F. Un service d'observation et de prévention permanent sera mis sur pied entre le $\mathrm{km} 2321$ et le $\mathrm{km} 2328$.

Les largeurs du chenal correspondant, respectirement, à la navigation à voie unique et à celle à deux voies, ont été fixés d'un commun accord avec les compagnies de navigation, et, compte tenu des difficultés exceptionnelles rencontrées sur ce parcours, ont également été sanctionnées par la Commission du Danube.

A l'aval de Vilshofen jusqu'à Passau, depuis l'aménagement de la retenue de Kachlet, la navigation dispose d'une profondeur d'eau de $2 \mathrm{~m}$ sur la quasi-totalité de la largeur de cette retenue, mème lorsque le plan d'eau se trouve à la plus basse cote navigable.

Des travaux de régularisation auraient de toute facon fini par s'imposer dans le parcours à l'aval de la centrale de Kachlet, afin d'y prévenir les tendances d'approfondissement du Danube à la suite de la régularisation de l'Inn, et par conséquent, de la diminution des débits solides de cette rivière. Il existait déjà autrefois des tronçons navigables à voie unique à divers endroits de ce parcours. La construction de la centrale de Jochenstein sur le Danube, au voisinage immédiat de la frontière germano-autrichienne, pendant les années 19521956 par la société «Donaukraftwerk Jochenstein A.G. » a réglé ces questions définitivement. L'aménagement de Jochenstein fut un projet réalisé en commun par l'Allemagne et l'Autriche. La largeur utile de $24 \mathrm{~m}$ et la longueur utile de $230 \mathrm{~m}$ des écluses doubles à Jochenstein correspondent aux dimensions des écluses de Kachlet. Le barrage comporte six passes de $24 \mathrm{~m}$ de large et des vannes présentant une hausse de $11,8 \mathrm{~m}$. La chute en régime de moyennes eaux, s'élève à $10,15 \mathrm{~m}$. La productivité d'une année moyenne des cinq groupes Kaplan de l'usine s'élève à 930 millions de $\mathrm{kWh}$. La réalisation de cette retenue a rendu superflus les travaux de régularisation du chenal d'étiage prévus dans des projets antérieurs pour le parcours de Jochenstein à Passau. D'après les dimensions-types fixées par la Commission du Danube pour les parcours allemand et autrichien, les parties du cours aménagées en retenues devront désormais présenter une profondeur navigable minimale de $2,70 \mathrm{~m}$ pour des fonds de gravier, et de $2,80 \mathrm{~m}$ pour des fonds rocheux (compte tenu du tirant d'eau du bateau de $1350 \mathrm{t}$ desservant le canal du Rhin à la Herne). Il est prévu de faire sauter les bosses rocheuses dans le fleuve au droit de la ville de Passau, afin d'en réduire la hauteur à 2,80 $\mathrm{m}$ au-dessous de la cote de retenue normale à Jochenstein, et ainsi de réaliser les conditions spécifiées par la Commission du Danube jusqu'à l'aval de la retenue de Kachlet.

Outre les travaux de la « Rhein-Main-Donau A.G. » déjà décrits, des mesures complémentares pour l'amélioration des conditions de navigation dans le Danube allemand ont été prises au cours des dernières années par l'Administration Fédérale des Eaux et de la Navigation. Après des travaux essentiels de dragage exécutés pendant les premières années qui suivirent la deuxième guerre mondiale, et en plus de la remise en état des berges sur de grandes longueurs, environ 3 millions de $m^{3}$ de gravier ont été dragués soit par l'Administration elle-mème, ou bien par des sous traitants. $40 \%$ du volume de gravier ainsi dragué a été soit remis en place dans le lit du fleuve, soit employé pour les travaux de construction. Il avait fallu draguer un volume aussi important de matériel afin de préserver le régime du fleuve, et de maintenir son chenal de navigation, puisque aucun dragage de gravier n'avait été effectué pendant la guerre. Afin d'éviter de favoriser l'approfondissement du fleuve, les travaux de dragage effectués de nos jours portent uniquement sur l'élimination de hauts-fonds susceptibles de gêner la navigation. Mais à mesure que l'aménagement du chenal d'étiage du fleuve se perfectionnera, ces bancs ne se présenteront plus que très localement. Des travaux de dragage importants seront encore nécessaires à deux endroits pendant assez longtemps, aux confluents de l'Isar et de l'Inn, ceci en tout cas jusqu'à ce que l'ensemble de ces deux rivières soit aménagé pour la production hydroélectrique.

Le Danube est relativement plus étroit et sinueux sur son parcours allemand que dans ses cours moyen et inférieur, mais le tracé de son chenal de navigation est généralement stable et ne varie que peu. Dans le but de permettre aux navigants de bien distinguer le chenal de navigation, surtout pendant la nuit, l'Administration a fait implanter au cours des dernières années deux centaines de balises de navigation réparties plus ou moins uniformément le long du parcours, parmi lesquelles figurent, dans le fleuve, des bouées munies de bandes réfléchissantes, sur le rivage, des signaux indicateurs de direction constitués de croix couchées, et une trentaine de feux clignotants dont le code varie suivant leur position en rive gauche ou en rive droite du fleuve. Les écluses de Kachlet et de Jochenstein comportent des feux de signalisation modernes. Il est prévu de renouveler l'ensemble de cette signalisation fluviale avant 1968 , afin de la rendre conforme aux recommandations de la Commission du Danube.

A la suite de l'aménagement du fleuve, et en particulier de l'exhaussement du plan d'eau provoqué par les retenues de Kachlet et de Jochenstein, le régime d'écoulement, et par conséquent celui des glaces, ont subi de profondes modifications sur l'ensemble du parcours à l'amont de Jochenstein. jusqu'à Vilshofen, soit sur une distance d'une cinquantaine de kilomètres. Il se forme presque chaque hiver une couche de glace sur les retenues, avec des empilements plus ou moins importants, selon les conditions météorologiques. Il est essentiel d'éliminer cette glace dans l'intérêt de l'écoulement et de la navigation; à cette fin l'Administration dispose de quatre brise-glace, et la Jochenstein A.G. de quatre. Mais grâce à la construction, dans la retenue de Kachlet, d'ouvrages protégeant la ville de Vilshofen contre les crues, et la réalisation actuellement en cours d'aménagements semblables dans la ville de Passau, il devrait être possible dorénavant d'éviter des dégâts importants dus aux glaces dans les retenues.

A la suite de l'aceroissement du trafic fluvial depuis la guerre, les ports de refuge disponibles à Regensburg, Deggendorf et Passau se sont montrés insuffisants. Malgré la place disponible à Regonsburg, pour environ 180 bateaux de différents tonnages, par exemple, il arrivait souvent que, 


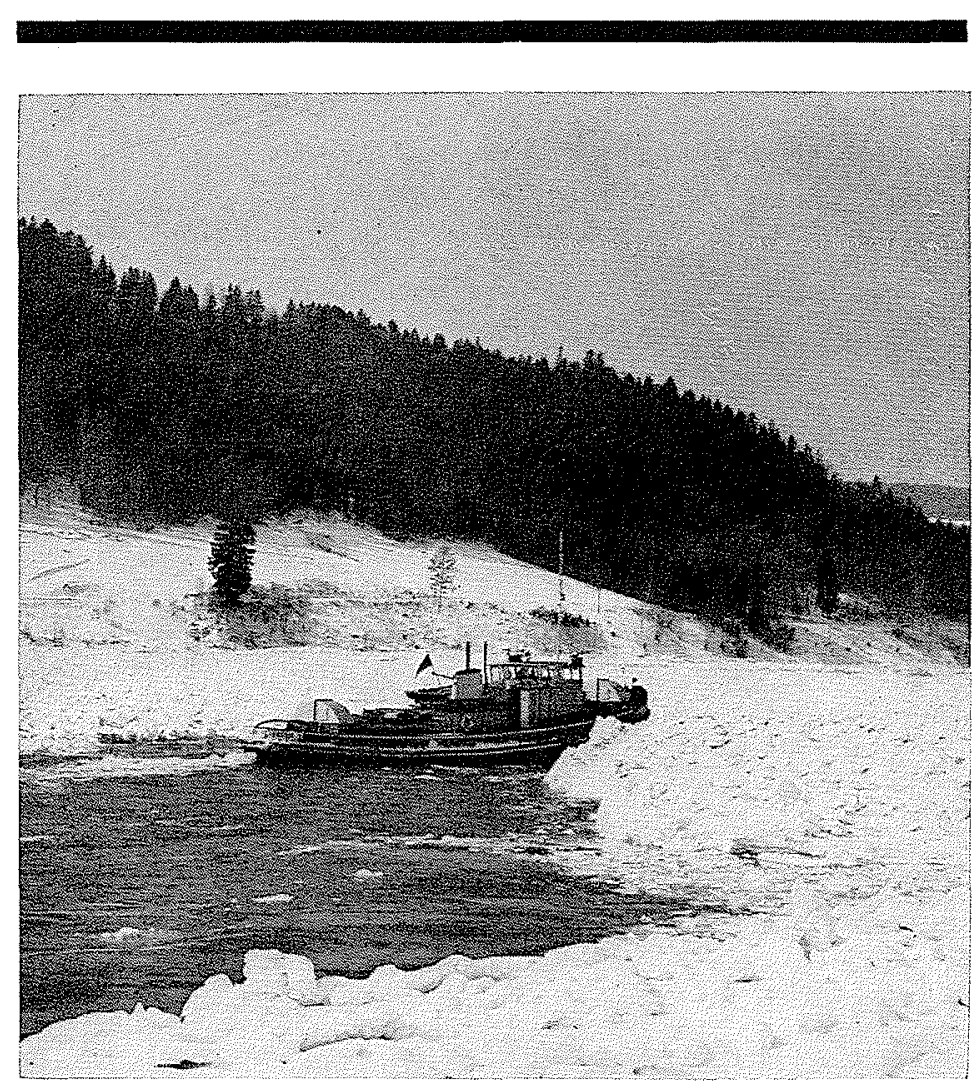

10/ Brise-glace travaillant dans la retenue de Jochenstein (cliché des archives de la centrale danubienne de Jochenstein AG, Passau).

Eisbrechereinsatz im Stauraum Jochenstein

(Bild aus dem Archiv der Donaukraftwerk Jochenstein AG, Passall).

pendant les périodes d'embâcle, des bateaux étaient obligés, soit de mouiller dans le fleuve même, ou dans l'entrée exposée du port, soit de se réfugier dans le bras mort du Danube à Bogen, où ils ne jouissaient que d'une protection toute relative. L'Administration a donc été obligée de construire un nouveau port de refuge, et puisque l'Administration bavardoise des Ports se trouvait également dans l'obligation d'agrandir le port de Regensburg afin de faire face à l'accroissement du trafic, il était tout à fait logique que ces deux administrations recherchassent une solution pouvant répondre à leurs besoins communs.

La zone riveraine au voisinage de Kreuznach, au $\mathrm{km} 2373$, paraissait toute indiquée pour la construction d'un nouveau port de refuge et de transbordement. L'Administration Fédérale des Eaux et de la Navigation a tout d'abord fait construire l'entrée du port et un bassin de virage (lesquels, grâce à la position favorable de la digue de protection contre les crues, constituaient déjà une zone abritée pour les bateaux), suivis par l'aménagement, par l'Administration bavardoise des Ports, du bassin de transbordement comportant des murs de quai verticaux. La longueur de ce port de refuge atteint environ $350 \mathrm{~m}$, son fond étant fixé à $2,50 \mathrm{~m}$ au-dessous de la plus basse cote navigable; les talus sont construits en pierre à la pente de $1: 2$, et comportent une butée de pied, ainsi qu'un perré de revêtement. Le port de refuge est concu pour servir également comme port de trans- bordement de secours. Le nouveau port de transbordement de Regensburg-Est mesure $400 \mathrm{~m}$ de long et $100 \mathrm{~m}$ de large; sa longueur pourrait toutefois être portée à $800 \mathrm{~m}$ en cas de nécessité.

\section{Problèmes d'aménagements futurs}

La régularisation du chenal d'étiage du Danube doit, en principe, ètre terminée en 1965. Il avait été prévu que les bateaux arrivant à Regensburg en provenance du canal Rhin-Main-Danube, dans lequel ils disposent d'un tirant d'eau de $2,50 \mathrm{~m}$, devraient, en période d'étiage, être allégés avant de continuer leur voyage sur le cours aval du Danube. Mais étant donné l'approfondissement progressif du fleuve à l'aval immédiat de Regensburg, les $2 \mathrm{~m}$ de profondeur d'eau prévus pour la régularisation du chenal d'étiage ne peuvent être assurés jusqu'à l'extrême amont du cours à Regensburg, c'est-à-dire à l'embarcadère situé dans le fleuve, et à l'intérieur du port Luitpold. Une barre rocheuse dans le fleuve (le «Hiltzler-Kachlet») juste à l'amont du pont de chemin de fer de Schwabelweis, a déjà été partiellement éliminée à l'explosif en 1959 afin d'assurer une profondeur d'eau de $2 \mathrm{~m}$ au plan d'eau correspondant à la plus basse cote navigable, mais par suite du creusement du lit du fleuve, cette profondeur n'atteint plus actuellement que $1,80 \mathrm{~m}$; or, compte tenu des hauteurs d'eau à l'amont, au débarcadère destiné aux transbordements, il n'est plus possible d'approfondir davantage aux explosifs. De même, le fleuve s'est approfondi à tel point que les bateaux ayant un tirant d'eau de 1,80 $\mathrm{m}$ ne peuvent plus accéder au port Luitpold à Regensburg (construit il y a une cinquantaine d'années) pendant les étiages, puisque les fonds dans ce port se trouvent maintenant à une cote relativement trop élevée, et ne peuvent être approfondis davantage. Les bateaux remontant le Danube peuvent, il est vrai, accéder au nouveau port, car il présente des profondeurs suffisantes, mais on ne pourra évidemment envisager, à long terme, que l'ancien port et l'embarcadère en rivière restent accessibles pendant chaque période d'étiage. Ces problèmes ne pourront être résolus définitivement que par l'aménagement d'une retenue à l'aval de Regensburg, et étant donné que, dans le temps, le lit du fleuve se creusera inévitablement à l'aval de cette retenue, d'autres retenues devront également être prévues par la suite. C'est d'ailleurs pour cette raison qu'il avait déjà été établi antérieurement un plan de canalisation pour l'ensemble du Danube allemand. Toutefois, cette canalisation présente l'inconvénient que, compte tenu de la faible pente entre Regensburg et Vilshofen (chute de $27 \mathrm{~m}$ seulement sur un parcours long de $130 \mathrm{~km}$ ) sa productivité hydroélectrique d'année moyenne ne pourrait atteindre qu'environ 600 millions de kWh; étant donné, en outre, que ce parcours traverse la région agricole la plus riche de toute la Bavière, et que, par conséquent, les exigences de l'agriculture, et la protection contre les crues, seront d'une importance capitale, ce projet serait extrêmement coûteux à réaliser. A titre estimatif, 
le montant de ces dépenses pourrait se situer autour de 500-600 millions de DM, représentant à peu près dix fois le coût des travaux de régularisation du canal d'étiage. Mais, étant donné, d'une part, que quatre retenues ont déjà été aménagées sur le seul parcours allemand et autrichien, et que, d'autre part, on prévoit la canalisation de l'ensemble du cours autrichien du fleuve (car ce n'est que par ce moyen que l'on pourra assurer pendant l'année entière la profondeur d'eau de $2,75 \mathrm{~m}$ nécessaire pour le bateau normalisé de $1350 \mathrm{t}$ ), il semble inévitable qu'un examen approfondi du problème de canalisation doive s'imposer à la longue.

\section{Zusammenfassung}

\section{Ausbau der deutschen Donau im Interesse der Landeskultur und der Schiffahrt Von Dipl. Ing. Franz Dobmayer *}

Im obersten rd. $194 \mathrm{~km}$ langen Abschnitt der deutschen Donau von Donaueschingen bis Ulm war die Mittelwasserkorrektion und der Hochwasserschutz, soweit ein solcher notwendig war, im wesentlichen schon um die Jahrhundertwende abgeschlossen.

Der Mittelwasserausbau im $174 \mathrm{~km}$ langen Abschnitt UIm-Kelheim konnte bis zum Jahre 1930 auf die ganze Länge von der bayerischen Staatsbauverwaltung zu Ende geführt werden. Hochwasserschutzanlagen auf größere zusammenhängende Strecken bestanden damals, zum Teil schon aus dem letzten Jahrhundert, im Abschnitt Dillingen- Donauwörth und unterhalb Ingolstadt; seit 1945 sind an verschiedenen Stellen noch Ergänzungen vorgenommen worden. Besondere Anlagen und Bauten für die Schiffahrt, die vor der Jahrhundertwende in diesem Abschnitt noch eine gewisse Bedeutung hatte, waren, von ganz wenigen Ausnahmen abgesehen, nicht notwendig gewesen.

Der Abschnitt Kelheim-Jochenstein, $213 \mathrm{~km}$, für den alle im Interesse der Schiffahrt notwendigen Ausbaumaßnahmen auf Grund des Staatsvertrags vom Jahre 1921 das Reich übernahm, war bis zu diesem Zeitpunkt zum überwiegenden Teil schon auf Mittelwasser korrigiert. Zusammenhängende Hochwasserdeiche gab es auf eine größere Länge vor dieser Zeit nur unterhalb Deggendorf. Eine grundlegende Hochwassersanierung für die landwirtschaftlich besonders wertwollen Ländereien im Straubinger, Deggendorfer und Pleintinger Becken wurde von der bayerischen Staatsbauverwaltung in den 30er Jahren begonnen und im letzten Jahrzehnt abgeschlossen. Alle diese Maßnahmen waren mit den entsprechenden Rücklaufdämmen an den einmündenden Seitenbächen und der gesamten Binnenentwässerung auf einen Schutz auch gegen die größten Hochwässer ausgerichtet.

Noch vor 1900 hatte sich auf diesem Donauabschnitt u.a. auch als Folge der günstigen Auswirkungen der Mittelwasserkorrektion, hauptsächlich aber mit dem Aufkommen der Dampfschiffahrt, ein reger Verkehr entwickest. Der bayerische Staat hat aus diesem Grunde um die Jahrhundertwende verschiedene Hafenanlagen in Regensburg, Deggendorf und Passau errichtet, die heute von der bayerischen Hafenverwaltung: betrieben werden. Der weitere Ausbau des Flusses für Zwecke der Schiffahrt, d.h., die Arbeiten der Niederwasserregulierung und die teilweise Kanalisierung der Strecke, wurden seit dem Jahre 1921 im Auftrag des Reiches bzw. des Bundes und des Staates Bayern von der RMD-AG durchgeführt. In den Jahren 1922-1927 wurde das Kachletwerk unterhalb Passau vornehmlich zur Verbesserung der Schiffahrtsverhältnisse in der besonders gefährlichen Felsstrecke Passau-Vilshofen errichtet. Die Niederwasserregulierung hatte beim niedrigsten Schiffahrtswasserstand, der z.Zt. durch einen Wasserstand von $116 \mathrm{~cm}$ am Pegel Schwabelweis charakterisiert ist, eine Fahrwassertiefe von $2 \mathrm{~m}$ mit den notwendigen Breiten zum Ziel. Nachdem die ersten Bauarbeiten mit verschiedenen Kurvenbegradigungen abgeschlossen waren, konnten von den $30 \mathrm{er}$ Jahren ab, auch die damals neuen $1000 \mathrm{t}$-Kähne mit einer Länge von ungefähr $70 \mathrm{~m}$ ohne Schwierigkeit verkehren, nachdem vorher überwiegend nur mit den kürzeren 600-700 t-Schiffen gefahren worden war. Die Niederwasserregulierungsarbeiten sind z.Zt. noch in vollem Gange und können etwa bis zum Jahre 1965 abgeschlossen werden. Der Schiffahrt wird dann an 308 Tagen im Jahr eine Fahrwassertiefe von $2 \mathrm{~m}$ zur Verfügung stehen.

Im letzten Jahrzehnt haben weitere Baumaßnahmen Verbesserungen gebracht: Mit der Stauanlage Jochenstein, die in den Jahren 1952-1956 von der Donaukraftwerk Jochenstein AG errichtet wurde, sind verschiedene einschiffige Strecken unterhalb Passau weggefallen. Die bayerische Hafenverwaltung und die Wasserstraßenverwaltung des Bundes haben mit Rücksicht auf den zunehmenden Verkehr unmittelbar unterhalb Regensburg, bei Kreuzhof, einen neuen Umschlags- und Sicherheitshafen errichtet. Weiter wurden durch umfangreiche Fahrwasserbaggerungen und eine ausreichende Fahrwasserbezeichnung die Schiffahrtsverhältnisse verbessert.

Neuerdings werden Ueberlegungen angestellt, wie und wann die Donaustrecke unterhalb Regensburg durchgehend kanalisiert werden soll.

* Direktor der Wasser- und Schiffahrtsdirektion, Regensburg. 